Original Article

\title{
Exogenously applied nutrients can improve the chickpea productivity under water stress conditions by modulating the antioxidant enzyme system
}

\author{
Nutrientes aplicados exogenamente melhoram a produtividade do grão-de-bico em \\ condições de estresse hídrico, modulando o sistema de enzimas antioxidantes
}

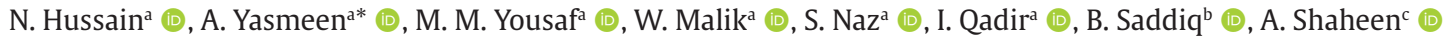 \\ and R. Iqbal ${ }^{\mathrm{b}}$ (D) \\ aBahauddin Zakariya University, Multan, Pakistan

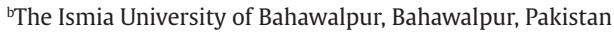

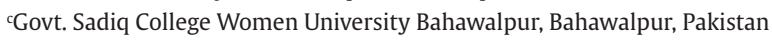

\begin{abstract}
Water stress is one of the major factor restricting the growth and development of chickpea plants by inducing various morphological and physiological changes. Therefore, the present research activity was designed to improve the chickpea productivity under water stress conditions by modulating antioxidant enzyme system. Experimental treatments comprised of two chickpea genotypes i.e. Bhakhar 2011 (drought tolerant) and DUSHT (drought sensitive), two water stress levels i.e. water stress at flowering stage and water stress at flowering + pod formation + grain filling stage including well watered (control) and three exogenous application of nutrients i.e. $\mathrm{KCl} 200 \mathrm{ppm}$, $\mathrm{MgCl}_{2}, 50 \mathrm{ppm}$ and $\mathrm{CaCl}_{2}, 10 \mathrm{mM}$ including distilled water (control). Results indicated that water stress at various growth stages adversely affects the growth, yield and quality attributes of both chickpea cultivars. Exogenous application of nutrients improved the growth, yield and antioxidant enzyme activities of both chickpea genotypes even under water stress conditions. However, superior results were obtained with foliar spray of potassium chloride on Bhakhar 2011 under well-watered conditions. Similarly, foliar spray of potassium chloride on chickpea cultivar Bhakhar 2011 cultivated under stress at flowering + pod formation + grain filling stage produced significantly higher contents of superoxide dismutase, peroxidase and catalase. These results suggests that the application of potassium chloride mitigates the adverse effects of water stress and enhanced tolerance in chickpea mainly due to higher antioxidant enzymes activity, demonstrating the protective measures of plant cells in stress conditions.
\end{abstract}

Keywords: antioxidant, leaf $\mathrm{K}^{+}$contents, catalase, potassium chloride, foliar spray.

\begin{abstract}
Resumo
O estresse hídrico é um dos principais fatores que restringem o crescimento e o desenvolvimento das plantas de grão-de-bico, induzindo várias alterações morfológicas e fisiológicas. Portanto, a presente atividade de pesquisa foi projetada para melhorar a produtividade do grão-de-bico em condições de estresse hídrico, por meio da modulação do sistema de enzimas antioxidantes. Tratamentos experimentais compostos de dois genótipos de grão-de-bico, ou seja, Bhakhar 2011 (tolerante à seca) e DUSHT (sensível à seca), dois níveis de estresse hídrico, ou seja, estresse hídrico na fase de floração e estresse hídrico na floração + formação de vagens + estágio de enchimento de grãos incluindo bem irrigado (controle) e três aplicações exógenas de nutrientes, ou seja, $\mathrm{KCl} 200$ ppm, MgCl2 50 ppm e CaCl2 $10 \mathrm{mM}$, incluindo água destilada (controle). Os resultados indicaram que o estresse hídrico em vários estágios de crescimento afeta negativamente os atributos de crescimento, rendimento e qualidade de ambas as cultivares de grão-de-bico. A aplicação exógena de nutrientes melhorou o crescimento, o rendimento e as atividades das enzimas antioxidantes de ambos os genótipos de grão- de-bico, mesmo em condições de estresse hídrico. No entanto, resultados superiores foram obtidos com pulverização foliar de cloreto de potássio em Bhakhar 2011, em condições bem irrigadas. Da mesma forma, a pulverização foliar de cloreto de potássio na cultivar de grão-de-bico Bhakhar 2011 cultivada sob estresse na fase de floração + formação de vagens + enchimento de grãos produziu teores significativamente maiores de superóxido dismutase, peroxidase e catalase. Esses resultados sugerem que a aplicação de cloreto de potássio atenua os efeitos adversos do estresse hídrico e aumenta a tolerância no grão-de-bico, principalmente em razão de mais atividade de enzimas antioxidantes, demonstrando as medidas protetoras das células vegetais em condições de estresse.
\end{abstract}

Palavras-chave: antioxidante, conteúdo foliar de $\mathrm{K}^{+}$, catalase, cloreto de potássio, spray foliar.

*e-mail: azra.yasmeen@bzu.edu.pk

Received: April 7, 2020 - Accepted: September 22, 2020

This is an Open Access article distributed under the terms of the Creative Commons Attribution License, which permits unrestricted use, distribution, and reproduction in any medium, provided the original work is properly cited. 


\section{Introduction}

Chickpea (Cicer arietinum L.) is a nutritionally important leguminous crop mainly cultivated on marginal lands in arid to semi-arid tropics (Jaleel et al., 2009). It plays an important role in human nutrition sectors in the developing world. It is ranked as a healthy food in many developed countries (Merga and Haji, 2019). Moreover, chickpea is also a crucial source of fodder and also fixes atmospheric nitrogen through symbiotic nitrogen fixation, hence plays a key role in enhancing and sustaining the overall productivity of the cropping systems (Khaitov et al., 2016). However, chickpea crop is facing severe water stress due to the unavailability of water, breaks-in precipitation associated with fewer rains at various growth stages causes the problem of erratic and terminal water stress and experiences up to 50\% yield loss (Sinha et al., 2019). Plants experience water stress either when the water supply to roots is interrupted or when the transpiration rate becomes very high. When plants are subjected to water stress conditions, show a lot of morpho-physiological and biochemical changes (Reddy and Raghavendra, 2006).

Plants under water stress conditions are unable to absorb sufficient nutrients from the soil causing nutrient deficiencies and as consequently reduced productivity. Exogenous application of essential nutrients mitigates these deficiencies and improves water stress tolerance by maintaining vital physiological processes. Among various nutrients, exogenous application of potassium stimulates a wide range of enzymatic systems, regulating water use efficiency, protein building, nitrogen uptake, photosynthesis and translocation of photosynthates into sink organs. Similarly, the application of calcium play role in controlling mechanisms, which stimulate the plant to become adopted to adversarial environmental conditions (Upadhyaya et al., 2011). Calcium performs an essential role in the activation of various defense mechanisms that are induced by water stress and $\mathrm{Ca}^{2+}$ signaling is mandatory for the acquisition of drought tolerance or resistance (Cousson, 2009). In addition to these nutrients, magnesium is also involved in numerous biochemical and physiological processes. It is the central core of the chlorophyll molecule in plant tissue and plays a role in the light absorbing the complex of chloroplasts and its contribution to photosynthetic fixation of carbon dioxide (Gerendás and Führs, 2013). It also participates in activation of many enzymes, for instance, phosphoenol pyruvate (PEP), glutathionsynthetase, carboxylase, kinases and phosphatases etc., (Bose et al., 2011). Magnesium is a crucial element for plant growth and development and plays an important role in plant defense mechanisms under water stress conditions (Cakmak and Yazici, 2010). A few studies were conducted to explore the role of calcium chloride, potassium chloride and magnesium chloride on the chickpea productivity under water stress conditions. It is hypothesized that foliar spray of calcium chloride, potassium chloride and magnesium chloride may positively affect the morpho-physiological processes of chickpea and enhanced the growth and productivity under water stress conditions. Keeping these facts in view, the present investigation was therefore undertaken to study the role of nutrients to mitigate the adverse effect of water stress on chickpea genotypes by modulating the antioxidant enzymes system.

\section{Materials and Methods}

\subsection{Experimental detail}

The present research activity was designed to improve the chickpea productivity under water stress conditions by modulating antioxidant enzyme system under agroecological conditions of Arid Zone Research Institute, Bahawalpur $\left(29.3871^{\circ} \mathrm{N}, 71.653^{\circ} \mathrm{E}\right)$ and Cholistan farm near Derawer $\left(28.19^{\circ} \mathrm{N}, 71.80^{\circ} \mathrm{E}\right)$ of Southern Punjab, Pakistan during Rabi 2014-15. Experimental treatments comprised of two chickpea genotypes i.e. Bhakhar 2011 (drought tolerant) and DUSHT (drought sensitive), two water stress levels i.e. water stress at flowering stage and water stress at flowering + pod formation + grain filling stage including well watered (control) and three exogenous application of nutrients i.e. $\mathrm{KCl} 200$ ppm (Bardhan et al., 2007), $\mathrm{MgCl}_{2}$, 50 ppm (Thalooth et al., 2006) and $\mathrm{CaCl}_{2,} 10 \mathrm{mM}$ (Xu et al., 2013) including distilled water (control). Foliar treatments were applied on $9^{\text {th }}$ January and $12^{\text {th }}$ February 2015 by using a hand sprayer. Field experiment was laid out in a Randomized Complete Block Design (RCBD) with factorial arrangement having three repeats.

\subsection{Soil analysis}

Prior to sowing, composite soil samples were collected from experimental areas at the depth of $0-30 \mathrm{~cm}$ and examined for physico-chemical features. The soil texture of experimental areas were loam and clay loam, having $\mathrm{pH}$ 8.2 and 7.9 , organic matter 0.77 and $0.93 \%$, EC 2.5 and $2.1 \mathrm{dS}$ $\mathrm{m}^{-1}$, total nitrogen 0.034 and $0.049 \%$, available phosphorus 6.3 and 4.5 ppm and exchangeable potassium 192 and 94 ppm in Bahawalpur and Cholistan, respectively.

Pre-soaking irrigation was applied before seedbed preparation. When the soil reached a workable moisture level, seedbed was prepared by cultivating the field twice with tractor-mounted cultivator each followed by planking during Rabi 2014-15. Seeds of both chickpea cultivars were sown by hand drill using $60 \mathrm{~kg} \mathrm{ha}^{-1}$ seed rate by keeping row to row distance of $25 \mathrm{~cm}$ and plant to plant distance $10 \mathrm{~cm}$ during last week of October 2014. Seeds were placed at 3 to $5 \mathrm{~cm}$ depth in each row. The seedlings were thinned out by maintaining one plant per hill at 15 days after sowing. The recommended dose of Urea @ $20 \mathrm{~kg} \mathrm{~N} \mathrm{ha}^{-1}$ and Di-ammonium phosphate (DAP) @ 22 kg P ha-1 was applied at the time of sowing. The crop was maintained free from weeds, diseases and pests by adopting appropriate plant protection measures. All other agronomic practices were kept normal for all the treatments. Data of different weather elements were collected from Meteorological Observatory, Arid Zone Agricultural Research Institute, Bahawalpur, Pakistan during the growing season. Data on average maximum and minimum temperature, average relative humidity and total rainfall were recorded on daily basis and averaged each month (Figure 1). 


\subsection{Data collection}

At 30 days after sowing, ten randomly selected plants were tagged to record the secondary branches per plant, number of pods per plant and 100 grain weight. Growth parameters were examined three times during growing seasons at both locations. Data regarding CGR was recorded by adopting the following Formula 1 of Watson (1952).

$$
C G R\left(g m^{-2} d a y^{-1}\right)=\left(W_{2}-W_{1}\right) /\left(T_{2}-T_{1}\right)
$$

whereas, $W_{1}$ and $W_{2}$ is the oven-dried weight at first and second sampling, $\mathrm{T}_{1}$ and $\mathrm{T}_{2}$ is the time of first and second sampling.

Relative growth rate (RGR) was calculated by using Equation 2 proposed by Radford (1967).

$$
R G R\left(g g^{-1} d a y^{-1}\right)=\left(L n W_{2}-\operatorname{LnW} 1\right) /\left(T_{2}-T_{1}\right)
$$

whereas, $\mathrm{Ln}=$ Natural $\log , \mathrm{W}_{1}$ and $\mathrm{W}_{2}$ is the oven-dried weight at first and second sampling, $\mathrm{T}_{1}$ and $\mathrm{T}_{2}$ is the time of first and second sampling.

The $\mathrm{K}^{+}$content in leaves were determined by flame photometer (Richards, 1954). Superoxide dismutase (IU $\mathrm{min}^{-1} \mathrm{mg}^{-1}$ protein) was calculated by adopting the methodology followed by (Giannopolitis and Ries, 1977). Moreover, peroxidase (IU $\mathrm{min}^{-1} \mathrm{mg}^{-1}$ protein) and catalase (IU $\mathrm{min}^{-1} \mathrm{mg}^{-1}$ protein) was calculated by adopting the procedure followed by Chance and Maehly (1955). $1 \mathrm{~m}^{2}$ from each experimental unit was harvested and threshed manually to record the grain yield per unit area. The crop was harvested on $4^{\text {th }}$ April 2015.

\subsection{Statistical analysis}

All the collected data was analyzed by using the MSTAT software. The Duncan's Multiple Range test (DMRt) was used to compare the differences among treatment means at $5 \%$ probability levels (Steel et al., 1997).

\section{Results}

\subsection{Crop growth rate $\left(\mathrm{g} \mathrm{m}^{-2}\right.$ day $\left.^{-1}\right)$}

Dry matter accumulation rate per unit land area is referred as crop growth rate (CGR). Data regarding CGR recorded at different growth stages were significantly affected by the interactive affects of chickpea genotypes, water stress at various growth stages and exogenous application of nutrients at both locations (Figure 1a and 1b). The CGR followed an increasing trend in the initial stages of crop growth and decreased thereafter. Exogenous application of potassium chloride on chickpea cultivar Bhakhar 2011 cultivated under well-watered condition produced significantly higher crop growth rate at all growth stages. Chickpea cultivar DUSHT cultivated under water stress at flowering + pod formation + grain filling stage produced minimum crop growth rate throughout the growing season.

\subsection{Relative growth rate $\left(\mathrm{g} \mathrm{g}^{-1}\right.$ day $\left.^{-1}\right)$}

Relative growth rate (RGR) is an obvious indicator of plant strategy with respect to productivity as related to environmental stress and disturbance regimes. RGR is the increase in size relative to the size of the plant present at the start of a given time interval. Data regarding RGR recorded at different growth stages were significantly affected by the interactive affects of chickpea genotypes, water stress at various growth stages and exogenous application of nutrients at both locations (Figure 2a and 2b). Exogenous application of potassium chloride on chickpea cultivar Bhakhar 2011 cultivated under well-watered condition produced significantly higher RGR at all growth stages. Chickpea cultivar DUSHT cultivated under water stress at flowering+ pod formation + grain filling stage produced minimum RGR throughout the growing season at both locations.

\subsection{Secondary branches per plant}

Secondary branches are mainly a genetic character and play an imperative role in improving the crop productivity.

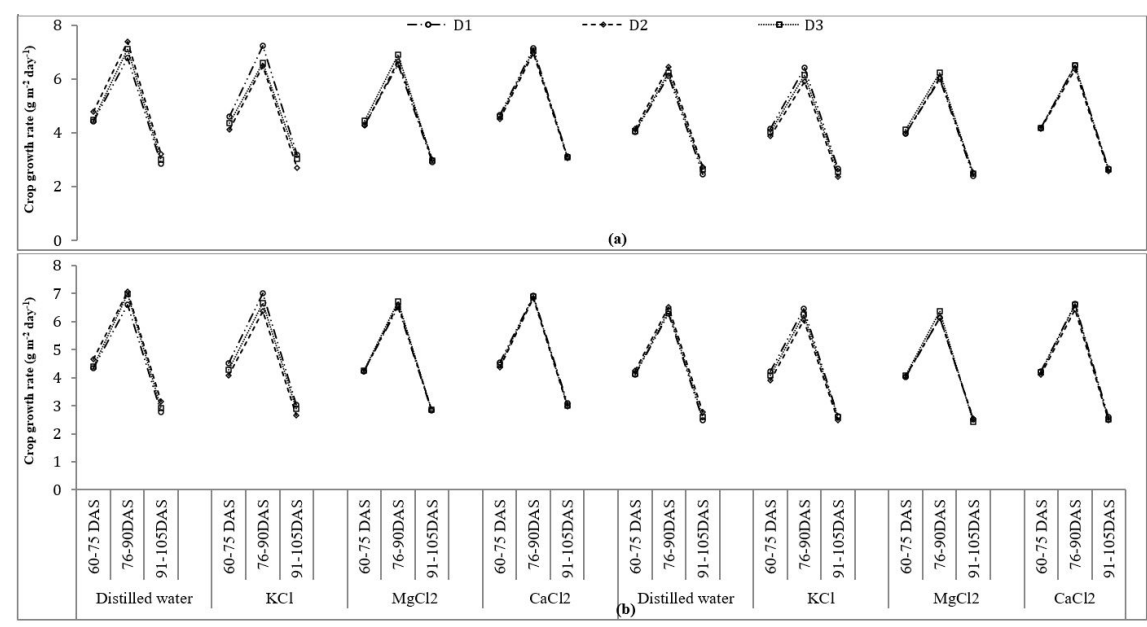

Figure 1. Effect of foliar application of nutrients on crop growth rate $\left(\mathrm{g} \mathrm{m}^{-2} \mathrm{day}^{-1}\right)$ of chickpea genotypes in Bahawalpur (a) and Cholistan (b). Whereas D1= well watered; D2=Drought at flowering+ pod formation + grain filling stage; D3= Drought at flowering stage; DAS= days after sowing. 


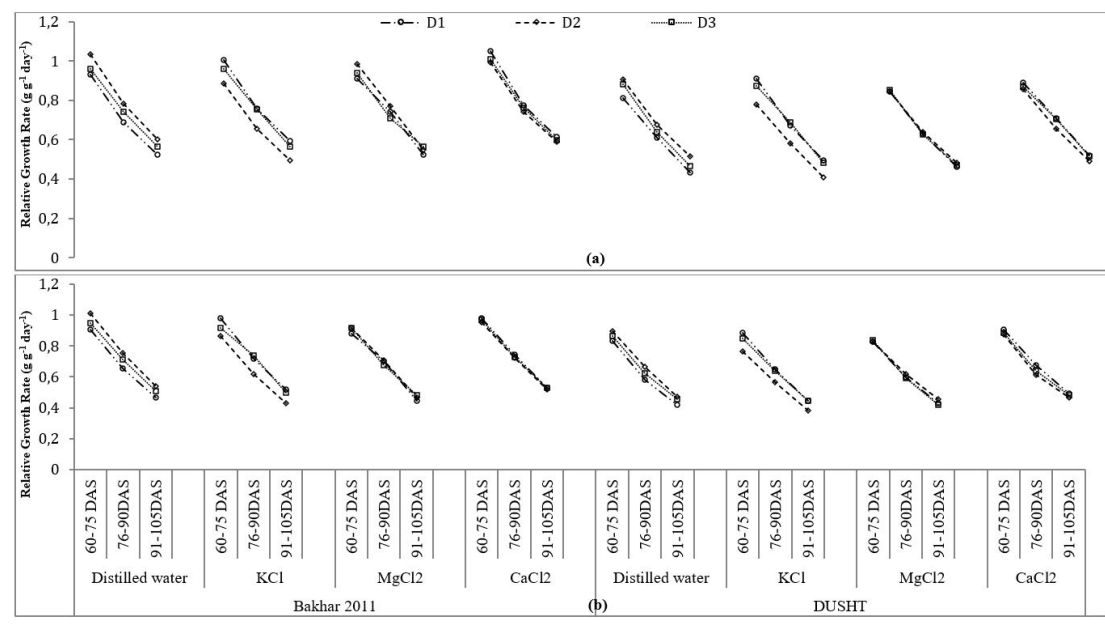

Figure 2. Effect of foliar application of nutrients on relative growth rate $\left(\mathrm{g} \mathrm{g}^{-1}\right.$ day $\left.^{-1}\right)$ of chickpea genotypes in Bahawalpur (a) and Cholistan (b). Whereas D1= well watered; D2= Drought at flowering+ pod formation + grain filling stage; D3= Drought at flowering stage; DAS= days after sowing.

Comparison of the treatment means showed that interaction among exogenous applications of nutrients, chickpea genotypes and water stress conditions at different growth stages significantly affected the number of secondary branches per plant. Foliar spray of nutrients significantly improved the secondary branches of both chickpea genotypes even under water stressed conditions. Exogenous application of potassium chloride on Bhakhar 2011 cultivated under well watered conditions produced maximum number of secondary branches per plant against the minimum was observed from same cultivar cultivated under water stress at flowering + pod formation + grain filling stage at both locations (Table 1 ).

\subsection{Number of pods per plant}

The results of analysis of variance values showed that interaction among exogenous applications of nutrients, chickpea cultivars and water stress conditions at different growth stages significantly affected the number of pods per plant (Table 1). Foliar spray of nutrients significantly improved the number of pods per plant of both chickpea genotypes even under water stressed conditions. Exogenous application of potassium chloride on Bhakhar 2011 cultivated under well watered conditions produced maximum number of pods per plant against the minimum was observed from DUSHT grown under water stress at flowering + pod formation + grain filling stage at both locations.

\subsection{0 grains weight (g)}

Among the various parameters contributing towards final yield of a crop, hundred-seed weight is of prime importance. Interaction among exogenous applications of nutrients, chickpea cultivars and water stress conditions at different growth stages significantly affected the hundred grains weight (Table 1). Exogenous application of calcium chloride and potassium chloride on Bhakhar 2011 cultivated under well watered conditions produced maximum hundred grains weight in Bahawalpur and Cholistan, respectively. Whereas the minimum hundred grains weight was observed from DUSHT cultivated under water stress at flowering + pod formation + grain filling stage.

\subsection{Biological yield ( $\left.\mathrm{kg} \mathrm{ha}^{-1}\right)$}

The biological yield refers to the total dry matter accumulation of a plant system. Interaction among exogenous applications of nutrients, chickpea genotypes and water stress at different growth stages significantly affected the biological yield (Table 2). Exogenous application of potassium chloride on Bhakhar 2011 cultivated under well watered conditions produced maximum biological yield against the minimum was observed from DUSHT grown under water stress at flowering + pod formation + grain filling stage at both locations.

\subsection{Grain yield ( $\left.\mathrm{kg} \mathrm{ha}^{-1}\right)$}

Crop yield is a measurement of the amount of agricultural production harvested per unit of land area. It can also refer to the actual grains generation from the chickpea plant. Interaction among exogenous applications of nutrients, chickpea cultivars and water stress conditions at different growth stages significantly affected the grain yield (Table 2). Foliar spray of nutrients significantly improved the grain yield of both chickpea genotypes even under water stressed conditions at both locations. Exogenous application of potassium chloride on Bhakhar 2011 cultivated under well watered conditions produced maximum grain yield against the minimum was observed from DUSHT grown under water stress at flowering + pod formation + grain filling stage at both locations.

\subsection{Leaf $\mathrm{K}^{+}$contents}

The results of analysis of variance values showed that interaction among exogenous applications of nutrients, chickpea cultivars and water stress conditions at different 
Table 1. Effect of foliar application of nutrients on secondary branches, pods per plant and 100 grains weight (g).

\begin{tabular}{|c|c|c|c|c|c|c|c|c|}
\hline \multirow{2}{*}{$\begin{array}{l}\text { Chickpea } \\
\text { genotypes }\end{array}$} & \multirow{2}{*}{$\begin{array}{c}\text { Water } \\
\text { stress level }\end{array}$} & \multirow{2}{*}{$\begin{array}{l}\text { Foliar } \\
\text { spray }\end{array}$} & \multicolumn{2}{|c|}{$\begin{array}{c}\text { Secondary branches per } \\
\text { plant }\end{array}$} & \multicolumn{2}{|c|}{ Number of pods per plant } & \multicolumn{2}{|c|}{100 grains weight $(\mathrm{g})$} \\
\hline & & & Bahawalpur & Cholistan & Bahawalpur & Cholistan & Bahawalpur & Cholistan \\
\hline \multirow[t]{12}{*}{$\begin{array}{c}\text { Bhakkar } \\
2011\end{array}$} & \multirow[t]{4}{*}{$\begin{array}{c}\text { Well } \\
\text { watered }\end{array}$} & $\begin{array}{l}\text { Distilled } \\
\text { water }\end{array}$ & $9.93 b c$ & $10.15 b c$ & 28.51af & $29.84 \mathrm{ad}$ & 203.48ad & 186.48ad \\
\hline & & $\mathrm{KCl}$ & $11.21 \mathrm{a}$ & $11.35 a$ & $32.37 a$ & $33.71 \mathrm{a}$ & 210.18ab & $193.83 \mathrm{a}$ \\
\hline & & $\mathrm{MgCl}_{2}$ & $10.47 b$ & $10.61 b$ & $29.56 \mathrm{ac}$ & $32.39 \mathrm{ab}$ & $205.50 \mathrm{ac}$ & $188.98 \mathrm{ad}$ \\
\hline & & $\mathrm{CaCl}_{2}$ & $10.51 b$ & $10.44 \mathrm{~b}$ & $30.08 \mathrm{ab}$ & $31.42 \mathrm{ac}$ & $212.12 \mathrm{a}$ & $191.21 \mathrm{ac}$ \\
\hline & \multirow{4}{*}{$\begin{array}{c}\text { Water } \\
\text { stress at } \\
\text { flowering+ } \\
\text { pod } \\
\text { formation + } \\
\text { grain filling } \\
\text { stage }\end{array}$} & $\begin{array}{l}\text { Distilled } \\
\text { water }\end{array}$ & $8.17 \mathrm{~g}$ & 8.34ij & $24.46 \mathrm{fg}$ & 22.62gi & 195.30be & $176.20 \mathrm{~cd}$ \\
\hline & & $\mathrm{KCl}$ & $8.693 \mathrm{dg}$ & 9.16dh & $27.17 \mathrm{bf}$ & $25.84 \mathrm{ci}$ & $204.43 \mathrm{ac}$ & $187.34 \mathrm{ad}$ \\
\hline & & $\mathrm{MgCl}_{2}$ & $8.43 \mathrm{fg}$ & $8.80 \mathrm{fi}$ & $25.79 \mathrm{cg}$ & $24.45 \mathrm{di}$ & 197.38ae & 187.75 ad \\
\hline & & $\mathrm{CaCl}_{2}$ & $8.42 \mathrm{fg}$ & 8.76gi & 25.17eg & $24.07 \mathrm{di}$ & 194.89be & 184.48 ad \\
\hline & \multirow{4}{*}{$\begin{array}{l}\text { Water } \\
\text { stress at } \\
\text { flowering } \\
\text { stage }\end{array}$} & $\begin{array}{l}\text { Distilled } \\
\text { water }\end{array}$ & $8.77 \mathrm{dg}$ & $9.17 \mathrm{dg}$ & $27.29 \mathrm{bf}$ & 26.62 bi & 201.54ae & 184.21ad \\
\hline & & $\mathrm{KCl}$ & $9.27 \mathrm{~cd}$ & $9.75 c d$ & $30.15 a b$ & 29.38 ae & $208.01 \mathrm{ac}$ & $192.68 \mathrm{ab}$ \\
\hline & & $\mathrm{MgCl}_{2}$ & 9.19de & $9.52 \mathrm{ce}$ & 28.93ae & 28.66af & 203.78ad & $187.67 \mathrm{ad}$ \\
\hline & & $\mathrm{CaCl}_{2}$ & $9.04 \mathrm{df}$ & $9.45 \mathrm{de}$ & 29.48ad & 28.48ag & 206.18ac & $189.00 \mathrm{ad}$ \\
\hline \multirow[t]{13}{*}{ DUSHT } & \multirow[t]{4}{*}{$\begin{array}{c}\text { Well } \\
\text { watered }\end{array}$} & $\begin{array}{l}\text { Distilled } \\
\text { water }\end{array}$ & $8.93 \mathrm{df}$ & $9.23 \mathrm{dg}$ & 25.17eg & $27.47 \mathrm{bh}$ & 197.38ae & 184.84ad \\
\hline & & $\mathrm{KCl}$ & $9.20 \mathrm{~d}$ & $9.58 \mathrm{~cd}$ & 28.52af & $30.77 \mathrm{ac}$ & 207.28ac & $190.85 \mathrm{ac}$ \\
\hline & & $\mathrm{MgCl}_{2}$ & $8.98 \mathrm{df}$ & 9.49de & $25.76 \mathrm{cg}$ & 28.75af & 202.38ae & $185.54 \mathrm{ad}$ \\
\hline & & $\mathrm{CaCl}_{2}$ & $9.02 \mathrm{df}$ & $9.43 \mathrm{df}$ & $26.18 b g$ & 28.09ah & 201.17ae & 185.72ad \\
\hline & \multirow{4}{*}{$\begin{array}{c}\text { Water } \\
\text { stress at } \\
\text { flowering+ } \\
\text { pod } \\
\text { formation + } \\
\text { grain filling } \\
\text { stage }\end{array}$} & $\begin{array}{l}\text { Distilled } \\
\text { water }\end{array}$ & $8.23 \mathrm{~g}$ & $8.06 \mathrm{j}$ & $22.41 \mathrm{~g}$ & $21.07 \mathrm{i}$ & $185.68 \mathrm{e}$ & $174.27 d$ \\
\hline & & $\mathrm{KCl}$ & $8.83 \mathrm{dg}$ & 8.66gj & $25.29 \mathrm{dg}$ & 23.41ei & 191.34ce & 181.33ad \\
\hline & & $\mathrm{MgCl}_{2}$ & 8.69dg & 8.52hj & 24.91eg & 22.41hi & 187.43de & $177.43 \mathrm{bd}$ \\
\hline & & $\mathrm{CaCl}_{2}$ & 8.51eg & $8.44 \mathrm{ij}$ & $24.37 \mathrm{fg}$ & $23.02 \mathrm{fi}$ & 193.69be & $176.99 \mathrm{~cd}$ \\
\hline & \multirow{4}{*}{$\begin{array}{c}\text { Water } \\
\text { stress at } \\
\text { flowering } \\
\text { stage }\end{array}$} & $\begin{array}{l}\text { Distilled } \\
\text { water }\end{array}$ & $8.44 \mathrm{fg}$ & 8.37ij & 24.93eg & $24.22 \mathrm{di}$ & 195.41ae & 183.84ad \\
\hline & & $\mathrm{KCl}$ & $9.06 \mathrm{df}$ & 8.93ei & $27.45 b f$ & 28.45ag & 204.83ac & $186.86 \mathrm{ad}$ \\
\hline & & $\mathrm{MgCl}_{2}$ & 8.76dg & 8.76gi & 26.13bg & 27.07bh & 203.06ad & $184.81 \mathrm{ad}$ \\
\hline & & $\mathrm{CaCl}_{2}$ & $8.86 \mathrm{dg}$ & 8.71gi & $26.83 \mathrm{bf}$ & 27.17 bh & 199.28ae & $184.32 \mathrm{ad}$ \\
\hline & LSD $0.05 p=$ & & 0.6906 & 0.6402 & 4.2079 & 5.9757 & 16.707 & 15.679 \\
\hline
\end{tabular}

LSD= Least significant difference.

growth stages significantly affected the leaf $\mathrm{K}^{+}$contents (Table 2). Exogenous application of potassium chloride on Bhakhar 2011 cultivated under well-watered conditions and water stress at flowering stage produced maximum leaf $\mathrm{K}^{+}$contents in Bahawalpur and Cholistan, respectively. Whereas, minimum leaf $\mathrm{K}^{+}$contents was observed from DUSHT grown under water stress at flowering + pod formation + grain filling stage at both locations.

\subsection{Superoxide dismutase (IU $\mathrm{min}^{-1} \mathrm{mg}^{-1}$ protein)}

Superoxide dismutase (SOD) is an enzyme that alternately catalyzes the dismutation of the superoxide $\left(\mathrm{O}_{2}^{-}\right)$radical into either ordinary molecular oxygen $\left(\mathrm{O}_{2}\right)$ or hydrogen peroxide $\left(\mathrm{H}_{2} \mathrm{O}_{2}\right)$. Interaction among exogenous applications of nutrients, chickpea cultivars and water stress conditions at different growth stages significantly affected the superoxide dismutase (Table 3). Exogenous application of potassium chloride on Bhakhar 2011 cultivated under water stress at flowering + pod formation + grain filling stage produced maximum superoxide dismutase against the minimum was observed from DUSHT grown under well watered conditions at both locations.

\subsection{Peroxidase (IU min-1 $\mathrm{mg}^{-1}$ protein)}

Interaction among exogenous applications of nutrients, chickpea cultivars and water stress conditions at different 
Table 2. Effect of foliar application of nutrients on biological yield $\left(\mathrm{kg} \mathrm{ha}^{-1}\right)$, grain yield $\left(\mathrm{kg} \mathrm{ha}^{-1}\right)$ and leaf $\mathrm{K}^{+}$contents.

\begin{tabular}{|c|c|c|c|c|c|c|c|c|}
\hline \multirow{2}{*}{$\begin{array}{l}\text { Chickpea } \\
\text { genotypes }\end{array}$} & \multirow{2}{*}{$\begin{array}{c}\text { Water } \\
\text { stress level }\end{array}$} & \multirow{2}{*}{$\begin{array}{l}\text { Foliar } \\
\text { spray }\end{array}$} & \multicolumn{2}{|c|}{ Biological yield $\left(\mathrm{kg} \mathrm{ha}^{-1}\right)$} & \multicolumn{2}{|c|}{ Grain yield $\left(\mathrm{kg} \mathrm{ha}^{-1}\right)$} & \multicolumn{2}{|c|}{ Leaf $\mathrm{K}^{+}$contents } \\
\hline & & & Bahawalpur & Cholistan & Bahawalpur & Cholistan & Bahawalpur & Cholistan \\
\hline \multirow[t]{12}{*}{$\begin{array}{c}\text { Bhakkar } \\
2011\end{array}$} & \multirow[t]{4}{*}{$\begin{array}{c}\text { Well } \\
\text { watered }\end{array}$} & $\begin{array}{c}\text { Distilled } \\
\text { water }\end{array}$ & 5693.1ad & 5793.1af & 1783.1af & 1792.1ad & 50.49ab & $47.15 \mathrm{dh}$ \\
\hline & & $\mathrm{KCl}$ & $6431.7 a$ & $6698.4 a$ & 2128.1a & $2108.8 \mathrm{a}$ & $56.57 a$ & $62.41 \mathrm{ab}$ \\
\hline & & $\mathrm{MgCl}_{2}$ & $5984.5 \mathrm{ac}$ & 6317.8 ac & $1868.4 \mathrm{ac}$ & $1976.7 \mathrm{ab}$ & $51.45 \mathrm{ab}$ & $50.70 \mathrm{ch}$ \\
\hline & & $\mathrm{CaCl}_{2}$ & 6197.7ab & 6497.7ab & 1923.3ab & 1970.3abc & 52.91ab & $48.25 \mathrm{ch}$ \\
\hline & \multirow{4}{*}{$\begin{array}{l}\text { Water } \\
\text { stress at } \\
\text { flowering+ } \\
\text { pod } \\
\text { formation } \\
+ \text { grain } \\
\text { filling } \\
\text { stage }\end{array}$} & $\begin{array}{c}\text { Distilled } \\
\text { water }\end{array}$ & $4765.6 \mathrm{ch}$ & 4065.6gi & 1405.3gi & $1214.5 \mathrm{fi}$ & $47.14 b$ & $42.08 \mathrm{gh}$ \\
\hline & & $\mathrm{KCl}$ & 5266.0af & 4799.3ci & 1629.5bh & $1467.3 \mathrm{ch}$ & 50.76ab & $57.063 \mathrm{ad}$ \\
\hline & & $\mathrm{MgCl}_{2}$ & 5141.7bg & 4375.0ei & $1533.0 \mathrm{ch}$ & 1382.0di & $48.12 \mathrm{ab}$ & $44.54 \mathrm{eh}$ \\
\hline & & $\mathrm{CaCl}_{2}$ & 4963.8bg & 4463.8di & $1503.1 \mathrm{ch}$ & $1368.3 \mathrm{di}$ & 51.64ab & $47.48 \mathrm{dh}$ \\
\hline & \multirow{4}{*}{$\begin{array}{c}\text { Water } \\
\text { stress at } \\
\text { flowering } \\
\text { stage }\end{array}$} & $\begin{array}{c}\text { Distilled } \\
\text { water }\end{array}$ & 5494.0ae & $4827.4 \mathrm{ci}$ & 1635.1bh & $1526.3 \mathrm{bh}$ & 51.28ab & $50.54 \mathrm{ch}$ \\
\hline & & $\mathrm{KCl}$ & $6027.5 \mathrm{ac}$ & 5827.5ae & 1976.2ab & $1802.4 \mathrm{ad}$ & 53.63ab & $63.14 \mathrm{a}$ \\
\hline & & $\mathrm{MgCl}_{2}$ & $5968.7 \mathrm{ac}$ & 5835.4ae & 1811.3ae & $1793.1 \mathrm{ad}$ & 51.06ab & $52.46 \mathrm{bg}$ \\
\hline & & $\mathrm{CaCl}_{2}$ & $5954.7 \mathrm{ac}$ & 5954.7ad & 1865.5ad & 1781.5 ae & $52.90 \mathrm{ab}$ & $51.27 \mathrm{ch}$ \\
\hline \multirow[t]{13}{*}{ DUSHT } & \multirow[t]{4}{*}{$\begin{array}{c}\text { Well } \\
\text { watered }\end{array}$} & $\begin{array}{c}\text { Distilled } \\
\text { water }\end{array}$ & 4547.6dh & 5214.2ah & $1420.5 \mathrm{fi}$ & $1608.3 \mathrm{ag}$ & $46.73 b$ & 43.98fh \\
\hline & & $\mathrm{KCl}$ & 5399.6af & 5732.9af & 1716.2bg & 1815.0 ad & 49.14ab & 55.11 ae \\
\hline & & $\mathrm{MgCl}_{2}$ & 4769.9ch & 5336.6 ag & $1489.7 \mathrm{dh}$ & 1671.6 af & 47.85ab & $45.54 \mathrm{eh}$ \\
\hline & & $\mathrm{CaCl}_{2}$ & 5241.5ag & 5274.8ah & $1543.2 \mathrm{ch}$ & 1662.5 af & $50.19 a b$ & $47.51 \mathrm{dh}$ \\
\hline & \multirow{4}{*}{$\begin{array}{l}\text { Water } \\
\text { stress at } \\
\text { flowering+ } \\
\text { pod } \\
\text { formation } \\
\text { + grain } \\
\text { filling } \\
\text { stage }\end{array}$} & $\begin{array}{c}\text { Distilled } \\
\text { water }\end{array}$ & $3644.4 \mathrm{~h}$ & $3344.4 \mathrm{i}$ & $1049.4 \mathrm{i}$ & $955.5 \mathrm{i}$ & $45.11 b$ & $41.05 \mathrm{~h}$ \\
\hline & & $\mathrm{KCl}$ & 4566.7dh & 3933.4gi & 1375.1gi & $1188.9 f i$ & 50.44ab & 53.42 af \\
\hline & & $\mathrm{MgCl}_{2}$ & 3991.2gh & 3791.2gi & 1268.9hi & 1097.3hi & $47.15 b$ & $45.30 \mathrm{eh}$ \\
\hline & & $\mathrm{CaCl}_{2}$ & 4311.5eh & 3744.8hi & 1269.4hi & $1127.8 \mathrm{gi}$ & 49.55ab & $48.10 \mathrm{ch}$ \\
\hline & \multirow{4}{*}{$\begin{array}{c}\text { Water } \\
\text { stress at } \\
\text { flowering } \\
\text { stage }\end{array}$} & $\begin{array}{c}\text { Distilled } \\
\text { water }\end{array}$ & 4185.5fh & 4252.1fi & 1319.6hi & 1281.1 ei & $47.10 \mathrm{~b}$ & $43.38 \mathrm{fh}$ \\
\hline & & $\mathrm{KCl}$ & $5057.1 \mathrm{bg}$ & 5323.8ag & 1616.6bh & 1674.0af & 50.73ab & $58.54 \mathrm{ac}$ \\
\hline & & $\mathrm{MgCl}_{2}$ & $4690.8 \mathrm{dh}$ & $4990.8 \mathrm{bh}$ & 1444.7eh & $1503.7 \mathrm{bh}$ & $46.50 \mathrm{~b}$ & $47.81 \mathrm{dh}$ \\
\hline & & $\mathrm{CaCl}_{2}$ & 4653.0cdh & 5253.0ah & 1445.7eh & $1594.0 \mathrm{bh}$ & 51.17ab & $49.73 \mathrm{ch}$ \\
\hline & LSD $0.05 p=$ & & 1262.7 & 1550.6 & 376.90 & 504.36 & 9.1396 & 10.586 \\
\hline
\end{tabular}

LSD= Least significant difference.

growth stages significantly affected the peroxidase (POD) (Table 3). Exogenous application of potassium chloride on Bhakhar 2011 cultivated under water stress at flowering + pod formation + grain filling stage produced maximum peroxidase against the minimum was observed from DUSHT grown under well watered conditions.

\subsection{Catalase (IU min $\mathrm{mg}^{-1}$ protein)}

Interaction among exogenous applications of nutrients, chickpea cultivars and water stress conditions at different growth stages significantly affected the catalase (CAT) (Table 3). Exogenous application of potassium chloride on
Bhakhar 2011 cultivated under water stress at flowering + pod formation + grain filling stage produced maximum catalase against the minimum was observed from DUSHT grown under well watered conditions at both locations.

\section{Discussion}

Water stress adversely affected chickpea growth and productivity by affecting the physiological and biochemical attributes. Exogenous application of mineral nutrients at $50 \%$ flowering and pod formation minimized the adverse affects of water stress by affecting the physiological 
Table 3. Effect of foliar application of nutrients on SOD (IU min ${ }^{-1} \mathrm{mg}^{-1}$ protein), POD (IU min-1 $\mathrm{mg}^{-1}$ protein) and CAT (IU min ${ }^{-1} \mathrm{mg}^{-1} \mathrm{protein}$.

\begin{tabular}{|c|c|c|c|c|c|c|c|c|}
\hline \multirow{2}{*}{$\begin{array}{l}\text { Chickpea } \\
\text { genotypes }\end{array}$} & \multirow{2}{*}{$\begin{array}{c}\text { Water stress } \\
\text { level }\end{array}$} & \multirow{2}{*}{$\begin{array}{l}\text { Foliar } \\
\text { spray }\end{array}$} & \multicolumn{2}{|c|}{$\begin{array}{l}\text { Superoxide dismutase } \\
\text { (IU } \text { min-1 }^{-1} \mathrm{mg}^{-1} \text { protein) }\end{array}$} & \multicolumn{2}{|c|}{$\begin{array}{c}\text { Peroxidase (IU min }{ }^{-1} \mathrm{mg} \text { - } \\
{ }^{1} \text { protein) }\end{array}$} & \multicolumn{2}{|c|}{$\begin{array}{c}\text { Catalase (IU min }{ }^{-1} \mathrm{mg}^{-} \\
{ }^{1} \text { protein) }\end{array}$} \\
\hline & & & Bahawalpur & Cholistan & Bahawalpur & Cholistan & Bahawalpur & Cholistan \\
\hline \multirow[t]{12}{*}{$\begin{array}{c}\text { Bhakkar } \\
2011\end{array}$} & \multirow[t]{4}{*}{ Well watered } & $\begin{array}{l}\text { Distilled } \\
\text { water }\end{array}$ & 70.67d & $64.01 \mathrm{e}$ & $6.84 \mathrm{e}$ & $6.46 \mathrm{~d}$ & 38.98de & 30.45 ef \\
\hline & & $\mathrm{KCl}$ & $81.45 d$ & 75.27 e & 7.10e & $7.30 \mathrm{~d}$ & $51.51 \mathrm{c}$ & $40.05 \mathrm{e}$ \\
\hline & & $\mathrm{MgCl}_{2}$ & $77.85 d$ & 70.64 e & $6.97 e$ & $7.07 \mathrm{~d}$ & 46.59ce & 37.77 ef \\
\hline & & $\mathrm{CaCl}_{2}$ & $80.92 d$ & 73.90 e & $7.03 e$ & $7.15 \mathrm{~d}$ & $48.64 c d$ & 38.90 ef \\
\hline & \multirow{4}{*}{$\begin{array}{l}\text { Water stress } \\
\text { at flowering+ } \\
\text { pod } \\
\text { formation }+ \\
\text { grain filling } \\
\quad \text { stage }\end{array}$} & $\begin{array}{l}\text { Distilled } \\
\text { water }\end{array}$ & $190.56 b c$ & $184.91 b c$ & $13.23 d$ & $15.04 \mathrm{c}$ & $88.65 b$ & 95.31 bc \\
\hline & & $\mathrm{KCl}$ & $216.62 a$ & $214.38 \mathrm{a}$ & 19.11a & $18.26 \mathrm{a}$ & $102.74 a$ & $110.96 \mathrm{a}$ \\
\hline & & $\mathrm{MgCl}_{2}$ & 197.89ab & $201.16 \mathrm{ab}$ & $17.14 \mathrm{bc}$ & $16.31 \mathrm{bc}$ & $93.80 \mathrm{ab}$ & $\begin{array}{c}102.88 \\
\mathrm{ac}\end{array}$ \\
\hline & & $\mathrm{CaCl}_{2}$ & 204.90ab & 206.60 a & 18.61ab & 17.97ab & $95.28 \mathrm{ab}$ & $\begin{array}{l}107.01 \\
\mathrm{ab}\end{array}$ \\
\hline & \multirow{4}{*}{$\begin{array}{c}\text { Water stress } \\
\text { at flowering } \\
\text { stage }\end{array}$} & $\begin{array}{l}\text { Distilled } \\
\text { water }\end{array}$ & 79.69d & $69.13 \mathrm{e}$ & $6.81 \mathrm{e}$ & $6.65 \mathrm{~d}$ & 42.55ce & 29.57ef \\
\hline & & $\mathrm{KCl}$ & $85.82 \mathrm{~d}$ & $74.15 \mathrm{e}$ & $7.23 e$ & $7.05 \mathrm{~d}$ & $52.29 c$ & $37.92 \mathrm{ef}$ \\
\hline & & $\mathrm{MgCl}_{2}$ & $77.92 d$ & 71.84 e & $6.83 e$ & $6.81 \mathrm{~d}$ & 45.70ce & $36.71 \mathrm{ef}$ \\
\hline & & $\mathrm{CaCl}_{2}$ & $79.13 d$ & $73.02 \mathrm{e}$ & $6.99 \mathrm{e}$ & $6.91 \mathrm{~d}$ & 47.48ce & $39.32 \mathrm{ef}$ \\
\hline \multirow[t]{13}{*}{ DUSHT } & \multirow[t]{4}{*}{ Well watered } & $\begin{array}{l}\text { Distilled } \\
\text { water }\end{array}$ & $66.64 d$ & 59.63 e & 5.47e & $6.04 \mathrm{~d}$ & $36.86 \mathrm{e}$ & $27.64 \mathrm{f}$ \\
\hline & & $\mathrm{KCl}$ & $78.83 d$ & $72.81 \mathrm{e}$ & $6.80 \mathrm{e}$ & $6.57 \mathrm{~d}$ & 43.56ce & $34.35 \mathrm{ef}$ \\
\hline & & $\mathrm{MgCl}_{2}$ & $76.63 d$ & $68.23 \mathrm{e}$ & $6.29 \mathrm{e}$ & $6.38 \mathrm{~d}$ & 41.69ce & $30.75 \mathrm{ef}$ \\
\hline & & $\mathrm{CaCl}_{2}$ & $78.20 d$ & $70.59 \mathrm{e}$ & $6.81 \mathrm{e}$ & $6.54 \mathrm{~d}$ & 43.03ce & 33.67ef \\
\hline & \multirow{4}{*}{$\begin{array}{l}\text { Water stress } \\
\text { at flowering+ } \\
\quad \text { pod } \\
\text { formation + } \\
\text { grain filling } \\
\quad \text { stage }\end{array}$} & $\begin{array}{l}\text { Distilled } \\
\text { water }\end{array}$ & $174.21 \mathrm{c}$ & $163.52 \mathrm{~d}$ & $16.49 c$ & $14.52 \mathrm{c}$ & $84.97 b$ & $81.36 \mathrm{~d}$ \\
\hline & & $\mathrm{KCl}$ & $192.83 b c$ & $186.53 \mathrm{bc}$ & $17.85 \mathrm{ac}$ & $16.39 \mathrm{bc}$ & 93.48ab & $98.91 \mathrm{ac}$ \\
\hline & & $\mathrm{MgCl}_{2}$ & $189.31 \mathrm{bc}$ & $175.75 \mathrm{~cd}$ & $16.52 c$ & $15.05 c$ & $87.52 b$ & $90.88 \mathrm{~cd}$ \\
\hline & & $\mathrm{CaCl}_{2}$ & $194.45 b$ & $182.43 \mathrm{c}$ & $17.48 \mathrm{ac}$ & $15.96 \mathrm{c}$ & 92.10ab & $93.57 \mathrm{c}$ \\
\hline & \multirow{4}{*}{$\begin{array}{c}\text { Water stress } \\
\text { at flowering } \\
\text { stage }\end{array}$} & $\begin{array}{l}\text { Distilled } \\
\text { water }\end{array}$ & $68.97 d$ & $63.33 \mathrm{e}$ & $6.30 \mathrm{e}$ & $6.12 \mathrm{~d}$ & 38.52de & 28.36 ef \\
\hline & & $\mathrm{KCl}$ & 77.16d & $70.88 \mathrm{e}$ & $6.84 \mathrm{e}$ & $6.68 \mathrm{~d}$ & 44.85ce & $34.70 \mathrm{ef}$ \\
\hline & & $\mathrm{MgCl}_{2}$ & $74.70 d$ & $69.33 \mathrm{e}$ & $6.43 e$ & $6.33 \mathrm{~d}$ & 41.72ce & 32.97ef \\
\hline & & $\mathrm{CaCl}_{2}$ & $78.16 d$ & 69.07 e & $6.67 e$ & $6.53 \mathrm{~d}$ & 43.94ce & 33.93ef \\
\hline & LSD $0.05 p=$ & & 20.008 & 17.190 & 1.7849 & 1.8719 & 10.846 & 12.159 \\
\hline
\end{tabular}

LSD $=$ Least significant difference.

parameters of chickpea genotypes. Foliar spray of potassium chloride on chickpea cultivar Bhakhar 2011 resulted in significantly higher growth attributes even under water stress conditions. It might be due to the fact that the exogenous application of potassium improves the photosynthetic efficiency and the succeeding carbohydrate synthesis and translocation that ultimately improve the crop growth attributes (Pettigrew, 2008). Potassium chloride plays a key role in the growth of the plants that utilize it as a source of macromolecules, which are required for normal growth and metabolic pathways of plants (Tariq et al., 2019).
Chickpea cultivars were cultivated under water stress conditions at different growth stages significantly reduced the number of secondary branches per plant at both locations. However, exogenous application of potassium chloride produced significantly higher number of branches per plants as compared to the other mineral nutrients by enhancing the photosynthetic efficiency and translocated the photo-assimilates to lateral meristems (El-Sabagh et al., 2017). It was observed that water stress at any specific growth stage or throughout the growing period significantly reduced the yield contributing attributes of chickpea cultivars (Nasri, 2005). Our results showed that water 
stress at flowering + pod formation + grain filling stage significantly reduced the number of pods per plant and 100 grains weight that ultimately reduced the biological and grain yield in the drought-sensitive cultivar (DUSHT) than in the resistant one (Bhakhar 2011). Reduction in yield components might be due to disturbing the plant water relations, fewer and uneconomical nutrients uptake, reduced leaf photosynthesis, dry matter partitioning, less accumulation of photo-assimilates and its remobilization from source to pods resulted in accelerate maturity with shriveled grains (Zhang et al., 2014). Exogenous application of mineral nutrients accelerates nutrient uptake and reduces the detrimental influences of water stress (Nasri, 2005). Among these mineral nutrients, potassium plays a crucial role in enhancing the plant tolerance under water stress situations. Potassium plays a vital role in number of physiological processes such as photosynthesis, translocation of photosynthates, activation of enzymes and balance of turgidity (Mengel and Kirkby, 2001). It was also observed that potassium played a key role in the osmotic adjustment of plants under water stress and improves the adaptability of the plant to stress conditions, which ultimately enhanced the productivity (Yadov, 2006).

Water stress has a strong detrimental influence on the chickpea plants and it reduced the uptake of mineral nutrients including potassium. Inadequate supply of potassium negatively influences the stomatal roles in plant body, ensuing in late stomatal closure and even the incomplete closure of the pores. In present investigation it was observed that exogenous application of potassium chloride significantly improved the potassium uptake even under water stress conditions. Sufficient supply of potassium can apparently improve the organic osmolyte production particularly under water stress situations (Zain et al., 2014). As plants are exposed to water stress, Pro accumulation plays an extremely defensive role and it is involved in osmotic adjustments in plants (Teixeira and Pereira, 2007).

Water stress reduced the photosynthetic efficiency of chickpea plants due to modifications in chlorophyll contents and destruction of the photosynthetic apparatus (Salekjalali et al., 2012). One of the imperative reasons that water stress weakened the photosynthetic abilities of plants is the interruption of the balance between the production of reactive oxygen species (ROS) and the antioxidant defense triggering accumulation of ROS that stimulates the oxidative stress to membrane lipids, proteins and other cellular components (Shen et al., 2010). Plants have evolved several molecular and physiological means to handle with negative influence of water stress (Chen and Murata, 2011). Therefore, plants have developed antioxidant defense system to protect themselves from ROS. This system draws on a series of antioxidant enzymes i.e. SOD, POD and CAT etc. (Lee et al., 2007). In present study, the results showed that exogenous application of potassium chloride on Bhakhar 2011 cultivated under water stress at flowering + pod formation + grain filling stage significantly improved the antioxidant enzymes against the minimum antioxidants were observed from DUSHT cultivated under well watered conditions. A higher activity of antioxidant enzymes under water stress conditions is the basic cause for scavenging $\mathrm{O}^{2-}$ radical to produce $\mathrm{H}_{2} \mathrm{O}_{2}$ (Bano et al., 2012). There are various stress reactions concerning $\mathrm{H}_{2} \mathrm{O}_{2}$, the homeostasis of $\mathrm{H}_{2} \mathrm{O}_{2}$ is mainly due to catalase scavenging and regulating functions. Thus, $\mathrm{H}_{2} \mathrm{O}_{2}$ accumulation was possibly the trigger of SOD activation. Zheng et al. (2008) observed that foliar spray of potassium detoxified the ROS through increased SOD, CAT, and POD enzyme activities under water stress conditions. Similar results were reported by Jan et al. (2017) they concluded that the antioxidant enzymes activities were increased with the exogenous application of potassium in stress conditions thus detoxified the ROS. Hence, the equilibrium between ROS and the anti-oxidative system is vital for endurance and adaptation to stress (Zhang et al., 2014).

\section{Conclusion}

The present results showed that exogenous application of potassium chloride on Bhakhar 2011 produced significantly higher growth, yield and antioxidant enzymes activities even under water stress conditions as compared to other treatments. These results suggests that the application of potassium chloride mitigates the adverse effects of water stress and enhanced tolerance in chickpea mainly due to higher antioxidant enzymes activity, demonstrating the protective measures of plant cells in stress conditions.

\section{References}

BANO, A., ULLAH, F. and NOSHEEN, A., 2012. Role of abscisic acid and drought stress on the activities of antioxidant enzymes in wheat. Plant, Soil and Environment, vol. 58, no. 4, pp. 181-185. http://dx.doi.org/10.17221/210/2011-PSE.

BARDHAN, K., KUMAR, V. and DHIMMAR, S.K., 2007. An evaluation of the potentiality of exogenous osmoprotectants mitigating water stress on chickpea. Journal of Agricultural Sciences, vol. 3, no. 2, pp. 67-74. http://dx.doi.org/10.4038/jas.v3i2.8101.

BOSE, J., BABOURINA, O. and RENGEL, Z., 2011. Role of magnesium in alleviation of aluminium toxicity in plants. Journal of Experimental Botany, vol. 62, no. 7, pp. 2251-2264. http://dx.doi. org/10.1093/jxb/erq456. PMid:21273333.

CAKMAK, I. and YAZICI, A.M., 2010. Magnesium: a forgotten element in crop production. Better Crops with Plant Food, vol. 94, pp. 23-25.

CHANCE, B. and MAEHLY, A.C., 1955. Assay of catalase and peroxidase. Methods in Enzymology, vol. 2, pp. 764-775. http:// dx.doi.org/10.1016/S0076-6879(55)02300-8.

CHEN, T.H. and MURATA, N., 2011. Glycinebetaine protects plants against abiotic stress: mechanisms and biotechnological applications. Plant, Cell E Environment, vol. 34, no. 1, pp. 1-20. http://dx.doi.org/10.1111/j.1365-3040.2010.02232.x. PMid:20946588.

COUSSON, A., 2009. Involvement of phospholipase C-independent calcium-mediated abscisic acid signaling during Arabidopsis response to drought. Biologia Plantarum, vol. 53, no. 1, pp. 5362. http://dx.doi.org/10.1007/s10535-009-0008-0.

EL-SABAGH, A., ABDELAAL, A.A. and BARUTCULAR, C., 2017. Impact of antioxidants supplementation on growth, yield and quality traits of canola (Brassica napus L.) under irrigation intervals in 
north Nile delta of Egypt. Journal of Experimental Biology and Agricultural Sciences, vol. 5, no. 2, pp. 163-172. http://dx.doi. org/10.18006/2017.5(2).163.172.

GERENDÁS, J. and FÜHRS, H., 2013. The significance of magnesium for crop quality. Plant and Soil, vol. 368, no. 1-2, pp. 101-128. http://dx.doi.org/10.1007/s11104-012-1555-2.

GIANNOPOLITIS, C.N. and RIES, S.K., 1977. Superoxide dismutases II. Purification and quantitative relationship with water-soluble protein in seedlings. Plant Physiology, vol. 59, no. 2, pp. 315318. http://dx.doi.org/10.1104/pp.59.2.315. PMid:16659840.

JALEEL, C., MANIVANNAN, P., WAHID, A., FAROOQ M., AL-JUBURI, H.J., SOMASUNDARAM, R. and PANNEERSELVAM, R., 2009. Drought stress In plants: a review on morphological characteristics and pigments composition. International Journal of Agriculture and Biology, vol. 11, pp. 100-105.

JAN, A.U., HADI, F., MIDRARULLAH., NAWAZ, M.A. and RAHMAN, K., 2017. Potassium and zinc increase tolerance to salt stress in wheat (Triticum aestivum L.). Plant Physiology and Biochemistry, vol. 116, pp. 139-149. http://dx.doi.org/10.1016/j. plaphy.2017.05.008. PMid:28558283.

KHAITOV, B., KURBONOV, A., ABDIEV, A. and ADILOV, M., 2016. Effect of chickpea in association with Rhizobium to crop productivity and soil fertility. Eurasian Journal of Soil Science, vol. 5, no. 2, pp. 105-112. http://dx.doi.org/10.18393/ejss.2016.2.105-112.

LEE, Y.-P., KIM, S.-H., BANG, J.-W., LEE, H.-S., KWAK, S.-S. and KWON, S.-Y., 2007. Enhanced tolerance to oxidative stress in transgenic tobacco plants expressing three antioxidant enzymes in chloroplasts. Plant Cell Reports, vol. 26, no. 5, pp. 591-598. http://dx.doi.org/10.1007/s00299-006-0253-z. PMid:17268803.

MENGEL, K. and KIRKBY, E.A., 2001. Principles of plant nutrition. 5th ed. Dordrecht: Kluwer Academic Publishers. http://dx.doi. org/10.1007/978-94-010-1009-2.

MERGA, B. and HAJI, J., 2019. Economic importance of chickpea: production, value, and world trade. Food \& Agriculture, vol. 5, no. 1, pp. 1615718. http://dx.doi.org/10.1080/23311932.20 19.1615718.

NASRI, M., 2005. Interaction of nutrient elements and drought stress in cultivars of Brassica napus. In: Proceedings of the 2 nd International Conference on Integrated Approaches to Sustain and Improve Plant Production Under Drought Stress, 2005, Rome, Italy. Rome: University of Rome "La Sapienza”, p. 109.

PETTIGREW, W.T., 2008. Potassium influences on yield and quality production for maize, wheat, soybean and cotton. Physiologia Plantarum, vol. 133, no. 4, pp. 670-681. http:// dx.doi.org/10.1111/j.1399-3054.2008.01073.x. PMid:18331406.

RADFORD, P.T., 1967. Growth analysis formulae, their use and abuse. Crop Science, vol. 8, no. 3, pp. 171-175. http://dx.doi. org/10.2135/cropsci1967.0011183X000700030001x.

REDDY, A.R. and RAGHAVENDRA, A.S., 2006. Photooxidative stress. In: K.V. MADHAVA RAO, A.S. RAGHAVENDRA and K.J. REDDY, eds. Physiology and molecular biology of stress tolerance in plants. The Nertherlands: Springer.

RICHARDS, L.A., 1954. Diagnosis and improvement of saline and alkali soils. Soil Science, vol. 78, no. 2, pp. 154. http://dx.doi. org/10.1097/00010694-195408000-00012.

SALEKJALALI, M., RAHEEM, H. and BEHBOUD, J., 2012. Effects of soil water shortages on the activity of antioxidant enzymes and the contents of chlorophylls and proteins in barley. AmericanEurasian Journal of Agricultural E' Environmental Sciences, vol. 12, pp. 57-63.
SHEN, J., XIE, K. and XIONG, L., 2010. Global expression profiling of rice microRNAs by one-tube stem-loop reverse transcription quantitative PCR revealed important roles of micro RNAs in abiotic stress responses. Molecular Genetics and Genomics, vol. 284, no. 6, pp. 477-488. http://dx.doi.org/10.1007/s00438-0100581-0. PMid:20941508.

SINHA, R., IRULAPPAN, V., MOHAN-RAJU, B., SUGANTHI, A. and SENTHIL-KUMAR, M., 2019. Impact of drought stress on simultaneously occurring pathogen infection in field-grown chickpea. Scientific Reports, vol. 9, no. 1, pp. 1-15. http://dx.doi. org/10.1038/s41598-019-41463-z. PMid:30944350.

STEEL, R.G.D., TORRIE, J.H. and DEEKEY, D.A., 1997. Principles and procedures of statistics: a biometrical approach. 3rd ed. New York: McGraw Hill Book, pp. 400-428.

TARIQ, F., BASIT, A., AHMAD, I., HAQ, S.I.U., ULLAH, Z., ALI, A., AHMED, J., RAHIM, A.A. and IQBAL, M.H., 2019. Comparative effects of potassium chloride $(\mathrm{KCl})$ as osmotic stressor on various growth parameters of Lycopersicon esculentum L. Pure and Applied Biology, vol. 8, no. 3, pp. 2065-2075. http://dx.doi. org/10.19045/bspab.2019.80151.

TEIXEIRA, J. and PEREIRA, S., 2007. High salinity and drought act on an organ-dependent manner on potato glutamine synthetase expression and accumulation. Environmental and Experimental Botany, vol. 60, no. 1, pp. 121-126. http://dx.doi.org/10.1016/j. envexpbot.2006.09.003.

THALOOTH, A., TAWFIK, M. and MOHAMED, H.M., 2006. A comparative study on the effect of foliar application of zinc, potassium and magnesium on growth, yield and some chemical constituents of mungbean plants grown under water stress conditions. World Journal of Agricultural Sciences, vol. 2, pp. 37-46.

UPADHYAYA, H., PANDA, S.K. and DUTTA, B.K., 2011. $\mathrm{CaCl}_{2}$ improves post drought recovery potential in Camellia sinensis (L) O Kuntze. Plant Cell Reports, vol. 30, no. 4, pp. 495-503. http://dx.doi. org/10.1007/s00299-010-0958-x. PMid:21153899.

WATSON, D.J., 1952. The physiological basis of variation in yield. Annals of Botany, vol. 4, pp. 101-105.

XU, C., LI, X. and ZHANG, L., 2013. The effect of calcium chloride on growth, photosynthesis, and antioxidant responses of Zoysia japonica under drought conditions. PLoS One, vol. 8, no. 7, pp. e68214. http://dx.doi.org/10.1371/journal.pone.0068214. PMid:23844172.

YADOV, D.V. 2006. Potassium nutrition of sugarcane. In: D.K. BENBI, M.S. BRAR and S.K. BANSAL, eds. Balanced fertilization for sustaining crop productivity. Horgen: Internat Potash Institute, pp. 275-288.

ZAIN, N.A.M., ISMAIL, M.R., PUTEH, A., MAHMOOD, M. and ISLAM, M.R., 2014. Drought tolerance and ion accumulation of rice following application of additional potassium fertilizer. Communications in Soil Science and Plant Analysis, vol. 45, no. 19, pp. 2502-2514. http://dx.doi.org/10.1080/00103624.201 4.932374 .

ZHANG, L., GAO, M., LI, S., ALVA, A.K. and ASHRAF, M., 2014. Potassium fertilization mitigates the adverse effects of drought on selected Zea mays cultivars. Turkish Journal of Botany, vol. 38, pp. 713723. http://dx.doi.org/10.3906/bot-1308-47.

ZHENG, Y., JIA, A., NING, T., XU, J., LI, Z. and JIANG, G., 2008. Potassium nitrate application alleviates sodium chloride stress in winter wheat cultivars differing in salt tolerance. Journal of Plant Physiology, vol. 165, no. 14, pp. 1455-1465. http://dx.doi. org/10.1016/j.jplph.2008.01.001. PMid:18313170. 